\title{
TOWARDS A PARAMETERIZATION METHOD FOR VIRTUAL SOFT TISSUES BASED ON PROPERTIES OF BIOLOGICAL TISSUE
}

\author{
Anderson Maciel, Ronan Boulic, Daniel Thalmann \\ Virtual Reality Lab, École Polytechnique Fédérale de Lausanne
}

\begin{abstract}
Realistic mechanical models of biological soft-tissues are a key issue to allow the implementation of reliable systems to aid on orthopedic diagnosis and surgery planning. We are working on a model of articulations based on deformation of connective tissues. In the present work we present several experiments towards the parameterization of a computer soft-tissues model from the elastic properties of real materials. We display our current results and draw comments on our experiments. Copyright $\odot 2003$ IFAC
\end{abstract}

Keywords: Musculoskeletal Systems, Articulations, Soft Tissues, Range of Motion, MRI, Surgery Planning.

\section{INTRODUCTION}

Biomechanics literature provides a number of mathematical models that approximate the behavior of bio-tissues and bio-structures. Most of them are typically conceptual. They are used in Biomechanics to understand the complex behavior of materials that are heterogeneous, anisotropic, viscoelastic, and which properties may drastically change due to environmental and use conditions. However, to be applied on graphical computer simulation of biomechanical systems, like the human musculoskeletal system, some model has to be converted and simplified to fit computer abilities.

We are currently working on a generalized approach towards functional modeling of human articulations". We plan to use such articulation model in medical applications to aid on diagnosis of joint disease and planning of surgical interventions. Our approach is based on a biomechanical model of the tissues present in the joint. This model relies on the mechanical and physical properties of biomaterials to provide correct motion, contact and deformation. The present work presents part of our biomechanical model. It deals with the important issue of

\footnotetext{
* This work was supported by the Swiss National Science Foundation in the framework of NCCR/CO-ME.
}

representing the mechanical properties of real soft tissues in their virtual models.

A number of computational methods to simulate soft tissues have been proposed. In this work we use a generalized mass-spring model, called molecular model (Jansson 2002), to simulate the behavior of cartilage and ligament. However, though mass-spring is a classical deformation approach, to set up the parameters of the model is not trivial. This problem is still more intricate if the behavior of a complex real material is intended to be represented.

The main goal of this work, thus, is to find a configuration of the elasticity coefficients of all springs in our molecular system, such that the elasticity of the whole piece of virtual tissue corresponds to the elasticity of the material it supposed to be made of. It is important to say that we are mostly interested now in the elastic part of the material behavior. Non-linear parts of the stressstrain curve will be discussed only as future work.

\section{BIO-TISSUES PROPERTIES}

The composition and behavior of bone, cartilage and ligament has been studied for many years. However, although we know much about these tissues, newer and better measurement techniques continuously change the available data. 


\subsection{Material Properties}

Ligament. Although significant advances have been made in the biology, biochemistry, and mechanics of soft tissue biomechanics, there is limited information available on in-vivo tissue mechanical characteristics and behavior. Without accurate values of such invivo information, extrapolations from animal and human insitu bone-ligament-bone testing to the function of intact human ligaments can not be made confidently. Currently, we know that ligaments are composite, anisotropic structures exhibiting nonlinear time and history-dependent viscoelastic properties. They have characteristics of strain rate sensitivity, stress relaxation, creep, and hysteresis. They exhibit significant time- and history-dependent viscoelastic properties. Ligaments are also temperature and age sensitive. See Fig. 1 for stressstrain relationship of ligament.

Cartilage. Articular cartilage, also called hyaline cartilage, is made of a multiphasic material with two major phases: a fluid phase composed by water (68$85 \%$ ) and electrolytes, and a solid phase composed by collagen fibrils (primarily type II collagen) (10$20 \%$ ), proteoglycans and other glycoproteins (5$10 \%$ ), and the chondrocytes (cartilaginous cells) (Mow, 1997). Properties of cartilage are anisotropic. The anisotropy results in part from the structural variations. Because of its structure cartilage is rather porous allowing fluid to move in and out of the tissue. When the tissue is subjected to a compressive stress, fluid flows out of the tissue. Fluid returns when the stress is removed. The mechanical properties of cartilage change with its fluid content, thus making it important to know the stress-strain history of the tissue to predict its load carrying capacity. The material properties also change with pathology. See Fig. 2 for stress-strain relationship of cartilage.

\subsection{Conclusion}

Biological tissues are materials of a very complex behavior. They present non-linear mechanical properties that vary from sample to sample, are dependent on structure and composition, and are time and history dependent. As a consequence of such wide set of variants, existent measured properties are not reliable and just barely describe the general behavior of these materials. Despite of that, specific situations can be delimited in which we are able to predict behavior from a reduced set of input parameter values. One of these situations is the linear elastic deformation of soft bio-tissues. Here is where this work puts its focus.

\section{DEFORMATION MODEL}

Approaches for modeling object deformation range from non-physical methods to methods based on continuum mechanics (Gibson, 1997). In this section, we focus on specific physically based approaches, which are used for modeling soft tissues. In this category, we present the two widest used classical approaches, mass-spring systems and finite element methods, and also our approach.

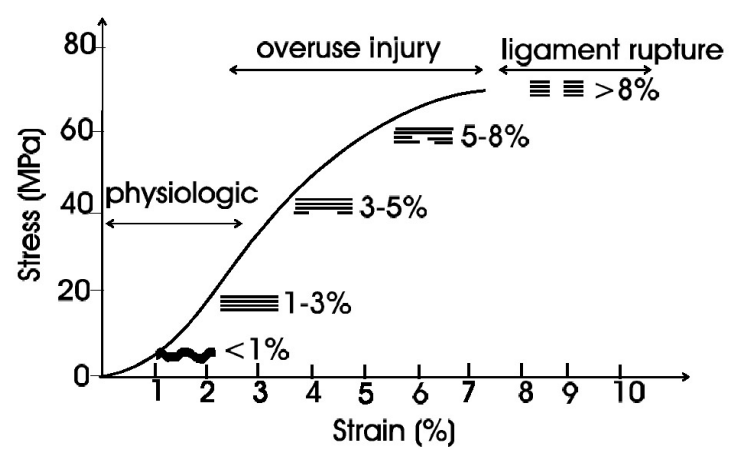

Fig. 1. Ligament stress-strain relationship. (Modified from Butler, 1984)

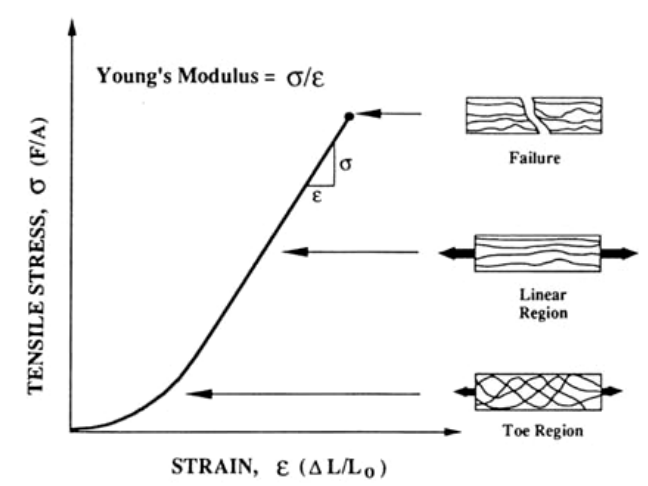

Fig. 2. Cartilage stress-strain curve. Young's modulus is defined by the slope of the linear region. (Adapted from Mow, 1991)

\subsection{Related Work}

Mass-spring systems. Mass-spring is a physically based technique that has been widely and effectively used for modeling deformable objects. An object is modeled as a collection of point masses connected by springs in a lattice structure. Springs connecting point masses exert forces on neighboring points when a mass is displaced from its rest position.

In biomechanical modeling, mass-spring systems were used by Nedel (1998) to simulate muscle deformation. Muscles were represented at two levels: action lines and muscle shape. This shape was deformed using a mass-spring mesh. Aubel (2001) used a similar approach with a multi-layered model based on physiological and anatomical considerations. Bourguignon and Cani (2000) proposed a model offering control of the isotropy or anisotropy of elastic material. The basic idea of their approach is to let the user define, everywhere in the object, mechanical characteristics of the material along a given number of axes corresponding to orientation of interest. All internal forces will be acting along these axes instead of acting along the mesh edges.

Mass-spring models are easy to construct, and both interactive and real-time simulations of mass-spring systems are possible even with desktop systems. Another well-known advantage is their ability to handle both large displacements and large deformations. However, mass-spring systems have 
some drawbacks. Since the model is tuned through its spring constants, good values for these constants are not always easy to derive from measured material properties. Furthermore, it is difficult to express certain constraints (like incompressibility and anisotropy) in a natural way.

Finite Elements Method. Whereas mass-spring models start with a discrete object model, more accurate physical models consider deformable objects as a continuum: solid bodies with mass and energies distributed throughout. Though models can be discrete or continuous, the method used for solving it is discrete. Finite element method is used to find an approximation for a continuous function that satisfies some equilibrium expression. In FEM, the continuum (object) is divided into elements joined at discrete node points. A function that solves the equilibrium equation is found for each element.

(Debunne et al., 2001) used a space and time adaptive level of detail, in combination with a large displacement strain tensor formulation. To solve the system, explicit FEM was used where each element is solved independently through a local approximation, which reduces computational time. Hirota et al. (2001) used FEM in simulation of mechanical contact between nonlinearly elastic objects. The mechanical system used as a case study was the Visible Human right knee joint and some of its surrounding bones, muscles, tendons and skin. The approach relied on a novel penalty finite element formulation based on the concept of material depth to compute skin, tendons and muscles deformation.

FEM provide a more realistic simulation than massspring methods but are computationally less efficient. In addition, the linear elastic theory used to derive the potential energy equation assumes small deformation of the object, which is true for materials such as metal. However, for soft biological material, objects dimensions can deform in large proportions so that the small deformation assumption no longer holds. Because of this change, the amount of computation required at each time is greatly increased.

\subsection{Molecules-based System}

Our approach to model soft-tissues is presented in this section. It is based in a work of Jansson et al., (2002) that was applied in computer aided design. Their work is founded in a generalized mass-spring model - which they call molecular model - where mass points are, in fact, spherical mass regions called molecules (Fig. 3). This approach is an analogy of the relationship of the real matter molecules with their neighborhood in a piece of material. Elastic forces are then established between molecules by a spring-like connection. In the present work we wish that properties of materials are taken into account to define the stiffness of such spring-like connections. However, though mass-spring is a classical deformation approach, to set up the parameters of the model is not trivial. This problem is still more intricate if the behavior of a complex real material is intended to be represented.
The force model. The model is described by two sets of elements: $E$, a set of spherical elements (molecules), and $C$, a set of connections between the elements in $E$ (Eq. 1).

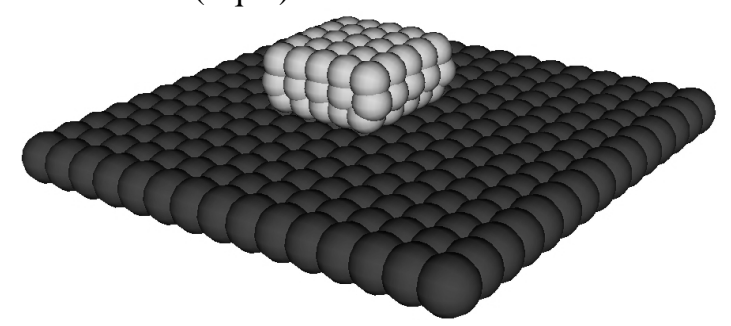

Fig. 3. Deformable tissues in contact.

$$
E=\left\{e_{1}, e_{2}, \cdots e_{n}\right\} ; C=\left\{C_{e_{1}}, C_{e_{2}}, \cdots C_{e_{n}}\right\} ; C_{e_{i}}=\left\{c_{1}, c_{2}, \cdots c_{m}\right\}
$$

The model's behavior is determined by the forces produced on each element of $E$ by each connection of $C$ and some external forces.

$$
\begin{aligned}
& \vec{F}_{e}=\vec{F}_{G}+\vec{F}_{L}+\vec{F}_{C}+\vec{F}_{\text {collisions }} \\
& \left\{\begin{array}{l}
\vec{F}_{G}=m_{e} \vec{g} \\
\vec{F}_{L}=-\Pi r_{e}^{2} \rho\left|\vec{V}_{e}\right|^{2} \frac{\vec{V}_{e}}{\left|\vec{V}_{e}\right|} \\
\vec{F}_{C}=\vec{F}_{b}+\vec{F}_{d}+\vec{F}_{f}
\end{array}\right.
\end{aligned}
$$

$F_{G}$ : gravity ( $m_{e}$ is the mass of $e$ and $g$ is the gravity acceleration);

$F_{L}$ : ambient viscous friction ( $r$ is the radius, $\rho$ is the medium density and $V$ is the velocity);

$F_{C}$ : connection forces, see Eq. 6.

$$
\begin{aligned}
& \vec{F}_{C}=\vec{F}_{b}+\vec{F}_{d}+\vec{F}_{f} \\
& \left(\vec{F}_{b}=\sum_{i=0}^{\mid C_{e}}-k_{c}\left(\left|\vec{P}_{e}-\vec{P}_{p}\right|-l_{c}\right) \frac{\vec{P}_{e}-\vec{P}_{p}}{\left|\vec{P}_{e}-\vec{P}_{p}\right|}\right. \\
& \vec{F}_{d}=\sum_{i=0}^{C_{e}}-b_{c}\left(\vec{V}_{\|}\right) \\
& \vec{V}_{\|}=\frac{\left(\vec{V}_{e}-\vec{V}_{p}\right) \cdot\left(\vec{P}_{e}-\vec{P}_{p}\right)}{\left|\vec{P}_{e}-\vec{P}_{p}\right|^{2}}\left(\vec{P}_{e}-\vec{P}_{p}\right) \\
& \vec{F}_{f}=\sum_{i=0}^{C_{e}}-\left|\mu_{e} \vec{F}_{N}\right| \frac{\vec{V} \perp}{|\vec{V} \perp|} \\
& \vec{F}_{N}=\vec{F}_{b}+\vec{F}_{d} \\
& \vec{V}_{\perp}=\left(\vec{V}_{e}-\vec{V}_{p}\right)-\vec{V}_{\|}
\end{aligned}
$$

$F_{b}$ : elasticity $\left(k_{c}\right.$ is the spring Hooke's constant, $l_{c}$ is the spring elongation, and $P_{e}$ and $P_{p}$ are the positions of the elements involved with connection $c$ );

$F_{d}$ : internal damping $\left(b_{c}\right.$ is the damping coefficient, $P$ and $V$ are respectively the positions and velocities of the elements involved with connection $c$ );

$F_{f}$ : sliding friction ( $\mu e$ is the friction constant for the element and $F_{N}$ is the force normal to friction direction).

\subsection{Setting up springs stiffness}

The rheological standard to define the elasticity of a material is Young's modulus. Young's modulus is a 
property of a material, not of an object. So it is independent of the object's shape. However, when you discretize an object by a set of springs, the stiffness of every spring must be proportional to the fraction of the volume of the object it represents.

Towards a generic method, able to calculate all spring constants in a system where the only information we have is the object's geometry, springs topology and Young's modulus of the material, we tested a number of hypotheses. These tests have been done on an implementation of our model where objects were deformed due to a pressure force - a homogenously distributed force on one face - applied onto them. To verify the correspondence between the deformation obtained with our model and the deformation the object should undergo with respect to its Young's modulus we used the following relation:

$$
E=\frac{F \cdot l_{0}}{\Delta l \cdot A},
$$

where $E$ is the Young's modulus, $F$ is the applied force, ? $l$ is the object's elongation, $l_{0}$ is the length of the object in rest conditions, and $A$ is the crosssectional area of the object.

So, we started by the simplest linear topology case and went through many variations until the generic random topology. Section 4 and conclusions should clarify the effectiveness of every method presented bellow.

Linear case. In the linear case, where all springs produce force in only one direction and one wants to measure elasticity in the same direction, one can easily calculate the constant $k$ of the springs directly from the geometrical information of the object and the Young's modulus of its material. This is done by using the eq. 14 where $k$ is the spring constant to be calculated. The problem of this very specific case is that its rectangular topology does not allow the construction of stable objects in two or three dimensions. Many solutions are valid as stable state (total elastic force equal to zero) and the situations of Fig. 5 may take place. To overtake the limitations of the linear case in terms of types of objects that can be represented, we tested two possibilities. The first using tetrahedral discretization and the second including diagonal springs to the rectangular topology. Both methods have allowed us to obtain stable objects. However, diagonal springs can make the number of springs grows up to $\left(n^{2}-n\right) / 2$, where $n$ is the number of molecules. This increases computation time.

$$
k=\frac{E A}{l_{0}}
$$

Tetrahedral regions. A straightforward path in order to reach object stability is creating a tetrahedral mesh of springs. With this method we get some stability and a method exists to calculate springs stiffness. Gelder (1998) presented a method to calculate stiffness for elastic edges of triangular meshes. His approach is based on the area of the/both triangles formed by the edge. He also proposed the extension of this approach to 3D, where volumes of neighboring tetrahedra are used to calculate the $k$ for an edge. The main drawback of this method is that it only works if the mesh of springs has a tetrahedral topology. $k$ is calculated as shown in Eq 15.

$$
k_{c}=\frac{E \sum_{e} \operatorname{vol}\left(T_{e}\right)}{|c|^{2}}
$$
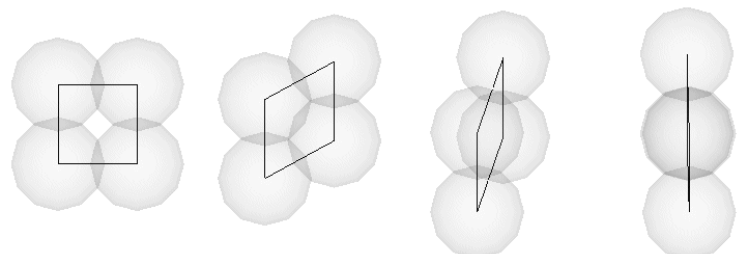

Fig. 5. These four situations are stable states with a rectangular topology. These and many other different relative positions between spheres are allowed for the same elongation of springs.

Diagonals on the planes. In order to avoid tetrahedral meshes, our first try in order to reach object stability was creating diagonal spring connections in every face of a rectangular mesh of springs. It offered the desired result but created a difficulty in terms of computing spring constants.

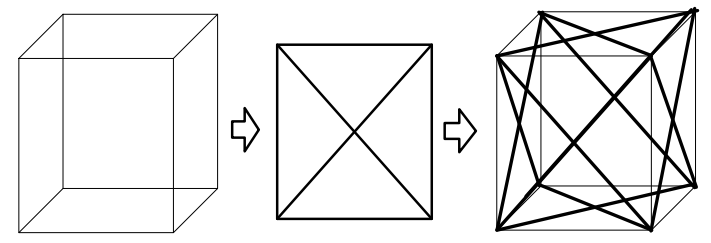

Fig. 6. Diagonals of faces.

If we use the standard linear method to compute spring constants, the diagonal springs will produce an extra force to linear movement that will causes elongation of the whole object to be shorter than it should. The object will become abnormally stiffer, and to avoid this some method should be developed to reduce springs $k$ 's. The straightforward solution is to divide all linearly calculated $k$ 's by a constant $C$. We tested arbitrary values to $C$ and concluded that even for the same topology, when other parameters change, such constant must change to preserve same elasticity. In addition, the problem persists when topology changes.

Diagonals on space. If we reduce the distance within which a connection must be created between two molecules, diagonals of parallelepiped are created in addition to rectangle's ones. In this case, other values to $C$ must be used to provide correct elasticity. Once we cannot work manually to find which constant best fulfils each new topology we have to deal with, we developed a method that automatically calculate new $k$ 's based on diagonal's angles.

Bringing the problem to $2 \mathrm{D}$ to make explanation easier, we see that force produced in vertical direction is increased by diagonals springs according to the angles between them. As we have right triangles in this topology, the relationship between a 
vertical connection and a diagonal one is proportional to cosine and the final $k$ is given by the relation:

$$
k_{f}=k_{0} \cos _{0}+k_{1} \cos _{1}+\ldots+k_{n} \cos _{n}
$$

where $k_{0}$ is the connection to which we are calculating $k$, and $k_{1}$ to $\mathrm{kn}$ are the connections that share a extremity with $k_{0} \cdot \cos _{i}$ is the cosine of the angle between connections 0 and $i$.

Considering a homogeneous object, all $k_{i} / l_{i}$ (where $l_{i}$ is the length of connection $i$ ) are equal. So, from this and Eq. 16 we deduce:

$$
k=\frac{k_{f}}{\left(l_{0}+l_{1} \cos _{1}+\ldots+l_{n} \cos _{n}\right)}
$$

This approximates the actual value of $k$. However, angles change along simulation and should be calculated for every iteration, which causes cost increase. Worse than that, the right triangles deform along simulation becoming arbitrary triangles, which invalids this method.

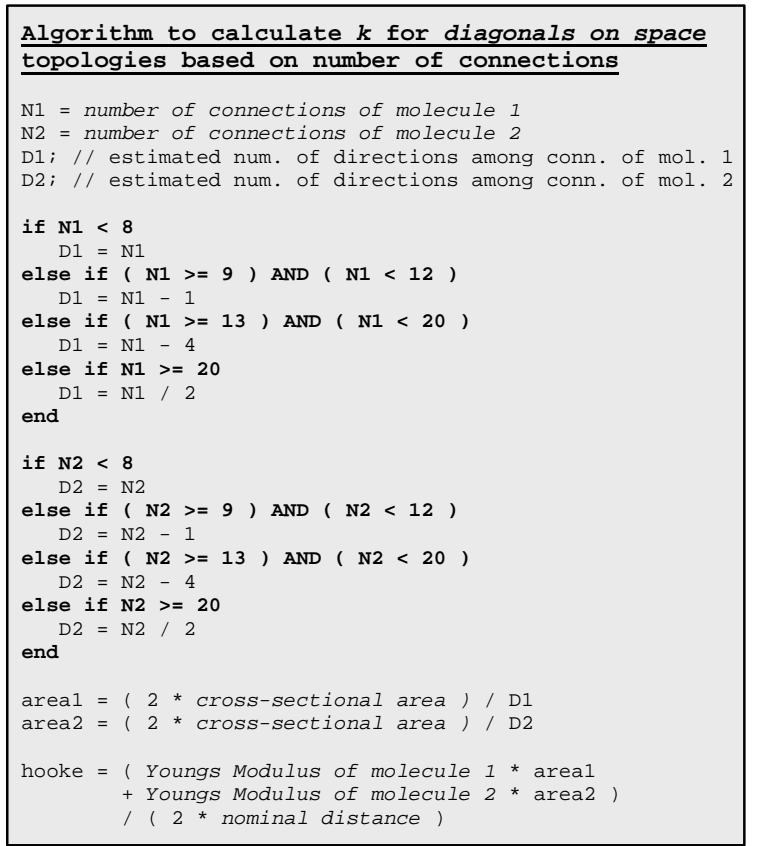

Fig. 7. Algorithm that calculates Hooke's constant based in our statistical method.

General case. As we have just seen, during deformation the molecules may change their positions in a way that diagonals' angles are no longer right angles. Besides, arbitrary initial topologies may happen where some angles are not right angles from the beginning. To handle these cases we developed a novel method to calculate spring constants. It is a statistical method inspired on general concepts of Quantum Mechanics that heuristically estimates $k$ 's reductions from the number of connections around a molecule. As the number of connections of a molecule increases, as small is the portion of the object's volume that each connection represents, and smaller must be its spring constant. So, though we do not calculate exactly the volume represented by a connection, we can probabilistically guess which topological case we have, just counting how many connections share a molecule. We considered the 3D case and stated the algorithm of Fig. 7 to calculate Hooke's constant for every connection of an object. This algorithm should present better results when larger and larger numbers of molecules are involved. It would happen because statistically, the finer the sampling the smaller the error.

\section{EXPERIMENTS}

We implemented the model described in section 3.3 in the form of a framework that will be used in a future work to develop medical applications. The code has been written in C++ on PC platform. In this implementation the forces are integrated along time to produce new molecules positions in a static simulation.

\subsection{Test Scenarios}

On the top of this application, a number of experiences have been performed to test the validity of the hypotheses presented in the section 3.3. Four deformable objects have been created which represent the same volume in the space (Fig. 8). Though they do not have exactly the same volume, the regions considered here have exactly the same vertical length and nearly the same cross sectional area. The vertical length of the considered volume, in the initial state is equal to $15 \mathrm{~mm}$ and its crosssectional area is around $9 \mathrm{~cm}^{2}$. The superior extremity of the objects is fixed and a tension force of $1 \mathrm{~N}$ is applied on the inferior extremity. The elasticity of the material is specified as $5000 \mathrm{~N} / \mathrm{m}^{2}(=$ $5 \mathrm{kPa}$ ). For each object we computed all methods to calculate $k$ presented in section 3.3. Table 1 compares the results obtained for Young's modulus when each method is used for calculation of $k$. The method based on tetrahedral regions has not been implemented here; we rely on the work of Gelder (1998). Later, to inspect the behavior of the methods when the number of molecules grows drastically, we created the situation of Fig. 15 where 1000 molecules and 7560 connections represent a volume of approximately $1 \mathrm{~m}^{3}$. The vertical length of the considered volume, in the initial state is equal to 86 $\mathrm{cm}$ and its cross-sectional area is around $1 \mathrm{~m}^{2}$. One extremity of the object is fixed and a tension force of $1000 \mathrm{~N}$ is applied on the other one. The elasticity of the material is specified as $105 \mathrm{~N} / \mathrm{m}^{2}(=100 \mathrm{kPa})$. The obtained results are in Table 2.

Table 1 Resultant elasticity obtained with the different methods for the 4 cases of Fig. 14. Shaded cells indicate correct values or with a small acceptable error.
(a)
(b)
(c)
(d)

\begin{tabular}{lccccc} 
Method & $\begin{array}{c}\text { Young's } \\
\mathbf{N} / \mathbf{m}^{2}\end{array}$ & $\begin{array}{c}\text { Young's } \\
\mathbf{N} / \mathbf{m}^{2}\end{array}$ & $\begin{array}{c}\text { Young's } \\
\mathbf{N} / \mathbf{m}^{2}\end{array}$ & $\begin{array}{c}\text { Young's } \\
\mathbf{N} / \mathbf{m}^{2}\end{array}$ \\
\hline Standard & 4994.50 & 4996.00 & 12465.71 & 20678.24 \\
Statistical & 10004.00 & 6098.30 & 3637.42 & 4904.84 \\
\hline $\begin{array}{l}\text { Angles } \\
\text { Angles } \\
\text { (update) }\end{array}$ & 4997.50 & 5000.50 & 4916.85 & 5903.88 \\
\hline
\end{tabular}


Table 2 Tension tests results for a cube of elasticity expected to be $10^{5} \mathrm{~N} / \mathrm{m}^{2}$.

\begin{tabular}{|c|c|c|}
\hline Method & $\begin{array}{c}\text { Elongation } \\
\quad(\mathbf{m m})\end{array}$ & $\begin{array}{c}\text { Young's } \\
\text { Modulus (N/m²) }\end{array}$ \\
\hline Standard & 2.300 & $373^{\prime} 913$ \\
\hline Statistical & 11.384 & $75^{\prime} 544$ \\
\hline Angles & 5.890 & $146^{\prime} 010$ \\
\hline$k / 3.75$ & 8.603 & $100^{\prime} 000$ \\
\hline
\end{tabular}

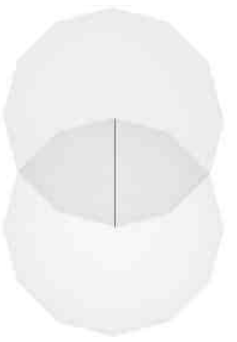

(a)

2 molecules

1 connection

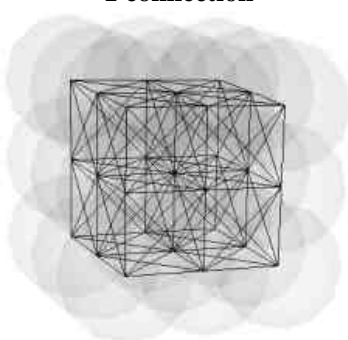

(c)

27 molecules

126 connections

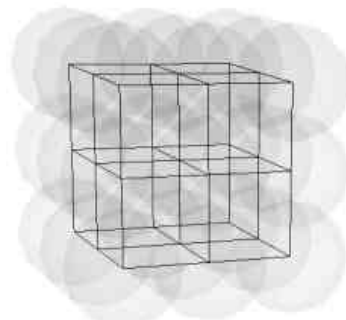

(b)

27 molecules

54 connections

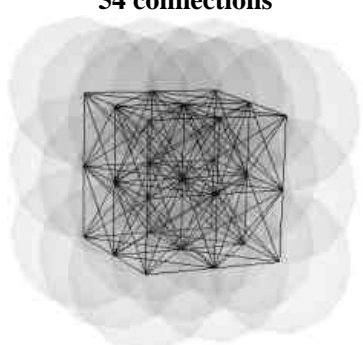

(d)

27 molecules 158 connections
Fig. 8. Scenarios being studied.

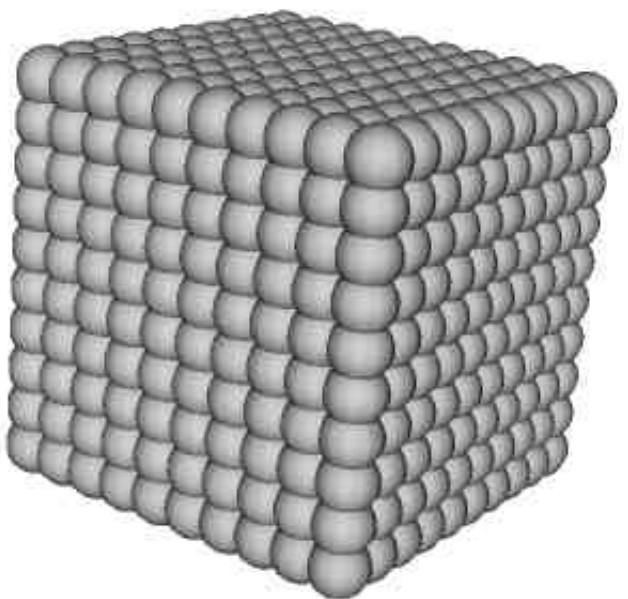

Fig. 15. Number of molecules and connections increased. 1000 molecules and 7560 connections.

\section{DISCUSSION AND CONCLUSIONS}

None of the methods tested here works unconditionally for any case. Standard method always works if mesh topology is rectangular, angles method if the diagonals form right triangles, and statistical method approximates the correct value in any case but usually gave objects softer than we expected. Both angles and statistical method can still be improved and we will keep working in this direction. We will also consider implementing tetrahedral method to apply it onto an intermediate tetrahedral mesh that can be constructed from arbitrary meshes, and then use the calculated values into the same arbitrary mesh. All our tests have been done applying tensile forces, but others, like shear test, must be done in order to validate a method for our purposes. Even if a spring configuration gives good results in tensile tests, we cannot affirm shear tests will also be correct. Finally, many important properties of tissues have not been taken into consideration in this work, like anisotropy and viscosity. Once these features become part of our deformation model, properties of materials will change along time, and any method used to configure our spring-like connections must take this factor into account to properly update all parameters during simulation.

\section{REFERENCES}

Aubel, A. and Thalmann, D. Interactive Modeling of Human Musculature. Computer Animation 2001, Seoul, Korea, November 7-8, 2001.

Bourguignon, D. and Cani, M-P. Controlling Anisotropy in Mass-Spring Systems. Computer Animation and Simulation 2000, pp. 113-123, August 21-22, Interlaken (Switzerland), 2000.

Butler, D.L. et al. Biomechanics of ligaments and tendons. Exercise and Sports Science Reviews, 6, 125-181, 1984.

Debunne, G. et al. Dynamic Real-Time Deformations Using Space \& Time Adaptive Sampling. Proceedings of SIGGRAPH 2001, Computer Graphics Proceedings, pp. 31-36, August 2001.

Gelder, A. Aproximate Simulation of Elastic Membranes by Triangulated Spring Meshes. In: Journal of Graphics Tools. v. 3, n. 2, pp. 21-42. A. K. Peters, Ltd. Natick, 1998.

Gibson, S. F. F. and Mirtich, B. A Survey of Deformable Modeling in Computer Graphics. Technical Report No. TR-97-19, Mitsubishi Electric Research Lab., Cambridge (Massachusetts), November 1997.

Hirota, G. et al. An Implicit Finite Element Method for Elastic Solids in Contact. Computer Animation 2001, Seoul (Korea), 2001.

Jansson, J. and Vergeest, J. S. M. A discrete mechanics model for deformable bodies. Computer-Aided Design. Amsterdam, 2002.

Mow V. C. et al. "Structure and Function of Articular Cartilage and Meniscus", Basic Orthopaedic Biomechanics, V.C. Mow and W.C. Hayes, Editors, Raven Press, New York, 1991.

Mow V. C. and Hayes W. C. Basic Orthopaedic Biomechanics. Second Edition. LippincottRaven Publishers, 1997. USA.

Nedel, L. P. and Thalmann, D. Real Time Muscle Deformations Using Mass-Spring Systems. Computer Graphics International 1998, pp. 156165, Hannover (Germany), June 1998. 